\title{
Talonavicular Fusion for Dorsal Subluxation of the Navicular in Resistant Clubfoot
}

\author{
Vineeta T. Swaroop MD, Dennis R. Wenger MD, \\ Scott J. Mubarak MD
}

Published online: 26 February 2009

(c) The Author(s) 2009. This article is published with open access at Springerlink.com

\begin{abstract}
Dorsal rotatory subluxation of the navicular, a common sequela of resistant surgically treated clubfeet, presents a challenging treatment problem. This subluxation typically progresses after posteromedial release. Patients develop a cavovarus foot deformity and complain of pain, gait problems, and difficulty with shoe wear. Previous attempts at soft tissue release and reduction have been largely unsuccessful. We reviewed 13 patients with dorsal rotatory subluxation of the navicular treated with talonavicular arthrodesis. The minimum followup after surgery was 6 months (average, 36 months; range, 6 to 93 months). At last followup 12 of 13 patients were symptom-free. The mean preoperative subluxation of $42 \%$ was reduced to a mean of $6 \%$ at last followup. We noted improvement in the talo-first metatarsal angle from an average of $18^{\circ}$ preoperatively to $8^{\circ}$ postoperatively. One patient treated by another surgeon with attempted talonavicular arthrodesis developed a nonunion; we observed no other complications. We believe talonavicular arthrodesis a reasonable option to correct the deformity and symptoms
\end{abstract}

Each author certifies that he or she has no commercial associations (eg, consultancies, stock ownership, equity interest, patent/licensing arrangements, etc) that might pose a conflict of interest in connection with the submitted article.

Each author certifies that his or her institution has approved the human protocol for this investigation and that all investigations were conducted in conformity with ethical principles of research, and that informed consent for participation in the study was obtained.

V. T. Swaroop

Rehabilitation Institute of Chicago, Chicago, IL, USA

D. R. Wenger, S. J. Mubarak ( $₫)$

Department of Orthopedic Surgery, Rady Children's Hospital, 3030 Children's Way, Ste 410, San Diego, CA 92123, USA

e-mail: pedsortho@chsd.org associated with dorsal rotatory subluxation of the navicular in a single surgical setting with a low complication rate.

Level of Evidence: Level IV, therapeutic study. See Guidelines for Authors for a complete description of levels of evidence.

\section{Introduction}

The residual or resistant clubfoot represents one of the most difficult treatment challenges for the pediatric orthopaedist. Often residual deformity is not obvious at the time of initial correction. With continued growth of the child, the persistent or undercorrected deformity becomes more apparent (refer to case seen in Fig. 2A-D). The resistant clubfoot must be carefully assessed, taking each deformity into account to plan appropriate treatment.

Dorsal rotatory subluxation of the navicular leading to residual cavovarus foot deformity is a common finding in a resistant postoperative clubfoot. Previous series have reported incidences varying from $7.1 \%$ (12 of 168 feet), to $34.9 \%$ ( 22 of 63 feet), to $54.2 \%$ (13 of 24 feet) of previously operated feet $[2,4,6]$. Barnett described the presentation of this deformity as a bimalleolar axis of less than $80^{\circ}$, internal foot progression angle, curved lateral border of the foot, shallow sinus tarsi, equinus, and rotation of the medial column of the forefoot [1]. This residual cavovarus can lead to several clinical problems including painful forefoot deformity, difficulty with shoe wear due to painful dorsal prominence, abnormal gait, and poor cosmesis [4]. As a result, dorsal rotatory subluxation of the navicular is associated with a high rate of revision surgery [4].

Surgical correction of symptomatic dorsal rotatory subluxation of the navicular is challenging. Both our own 
experience and that reported by others has shown repeat conventional soft tissue release results in persistence of the subluxation [1].

This retrospective review was performed for the following purposes: (1) to describe the outcomes of talonavicular arthrodesis with respect to symptomatic relief of dorsal rotatory subluxation of the navicular, (2) to assess correction of radiographic deformity, and (3) to identify associated complications.

\section{Materials and Methods}

We retrospectively reviewed the medical records of all 13 patients (13 feet) who underwent talonavicular arthrodesis to treat symptomatic dorsal rotatory subluxation of the navicular in resistant clubfoot between 1999 and 2007. Symptoms necessitating surgical treatment included pain, difficulty with shoe wear, gait abnormality, and unacceptable cosmesis. There were 11 boys and two girls. The average age at the time of surgery was $11 \pm 6$ years (range, $6 \pm 6$ years to $17 \pm 6$ years). Twelve of the 13 patients had idiopathic clubfoot. The remaining patient had underlying spastic quadriplegia and developed a clubfoot deformity after an iatrogenic nerve injury prior to presentation at our institution. All children were treated with previous complete posteromedial clubfoot release, and one patient had a previous attempt at talonavicular fusion performed by another surgeon. Patients were followed until radiographs demonstrated solid fusion mass at the arthrodesis site. The minimum followup after surgery was 6 months (average, 35.5 months; range, 6 to 93 months). No patients were lost to followup. We obtained prior institutional review board approval for reviewing the records.

The two senior authors (SJM and DRW) performed all surgery. The surgical technique began with an incision made on the medial aspect of the foot beginning approximately $1 \mathrm{~cm}$ below the medial malleolus and extending past the tuberosity of the navicular toward the first metatarsal. The insertion of the anterior tibial tendon was identified and preserved. The posterior tibial tendon was identified in the proximal aspect of the wound and traced distally to its insertion on the beak of the navicular. It was dissected from its sheath and released from the navicular and later repaired. The talonavicular joint capsule was opened widely to expose the articular surfaces of the talar head and proximal navicular. The soft tissues and joint capsule were elevated from the navicular in order to allow reduction onto the talus. Any scar tissue limiting this reduction dorsally was excised. Occasionally the naviculocuneiform joint was opened as well to facilitate accurate reduction of the navicular.
Next all cartilage was denuded from the talar head and from the articular surface of the navicular using a rongeur and curettes. One must preserve the subchondral bone to prevent excessive shortening of the medial column but also thoroughly remove all articular cartilage in order to obtain the desired fusion. After realigning the navicular in a plantar direction onto the talus, it was held in a reduced position using Kirschner wires placed in a distal to proximal fashion into the body of the talus. Adequacy of reduction was verified using intraoperative imaging to confirm restoration of a normal lateral talo-first metatarsal angle. Compression and fixation were achieved using one or two 4-mm or 4.5-mm partly threaded cannulated cancellous screws. Morselized local autograft from the medial navicular was then placed at the fusion site. Osteotomies of the cuboid or calcaneus were occasionally necessary to facilitate the reduction and improve the position of the foot. The arthrodesis was performed with one screw in seven cases, two screws in four cases, and two Kirschner wires in two cases. In most cases utilizing one screw, fixation was augmented with a Kirschner wire. In seven cases, morselized local autograft was placed at the fusion site. Additional procedures performed in the same setting included sliding calcaneal osteotomy (five cases), closingwedge cuboid osteotomy (three cases), cuneiform osteotomy (one case), first metatarsal osteotomy (two cases), Vulpius-type tendo-Achilles lengthening (three cases), phalangeal osteotomy (two cases), individual toe flexor tenotomies (five cases), anterior tibial tendon transfer (two cases), distal tibia/fibula varus osteotomy (one case), calcaneocuboid fusion (three cases), and naviculocuneiform fusion (one case). The wound was closed in layers and a well-molded short leg cast was placed, which was split and spread for swelling.

Patients were admitted to the hospital overnight for monitoring and pain control. After discharge patients were seen for followup at 1 week for cast overwrap. They were maintained non-weight bearing in a short-leg cast for 4 weeks. At that point radiographs were obtained. If sufficient healing was observed, patients were then placed back into a short-leg walking cast and allowed to progressively weight bear as tolerated in the cast for an additional 4 weeks.

From the medical records we recorded previous treatment for clubfoot, age at time of talonavicular arthrodesis, details of surgical procedure including any simultaneously performed procedures, complications, and need for further treatment.

We obtained pre- and postoperative standing anteroposterior and lateral radiographs on all patients. One of us (VTS) not involved in the treatment assessed the percent subluxation of the navicular on the lateral radiograph, calculated as the vertical dorsal displacement of the 
superior pole of the navicular above the line connecting the dorsal aspect of the head of the talus and the medial cuneiform [4] divided by the total vertical length of the navicular. The lateral talo-first metatarsal angle and AP talo-first metatarsal angle were also assessed. Postoperative radiographs were also assessed for adequacy of bony fusion mass as demonstrated by lack of a visible joint space between the talus and navicular with presence of spanning bone as well as preservation of postoperative correction.

\section{Results}

At final followup, 12 of 13 patients were symptom-free. One patient, a college baseball player, complained of lateral foot pain we attributed to calcaneofibular impingement. Otherwise all patients demonstrated plantigrade foot position. Three patients had returned to sports; the records contained no notes of other limitations from the foot and no difficulty with shoe wear.

The average preoperative percent subluxation of the navicular was $42 \%$ (range, 5\%-76\%). Postoperatively the average percent subluxation decreased to $6 \%$ (range, 0$33 \%$ ). The lateral talo-first metatarsal angle improved from an average of $18^{\circ}$ (range, $5^{\circ}-40^{\circ}$ ) preoperatively to $8^{\circ}$

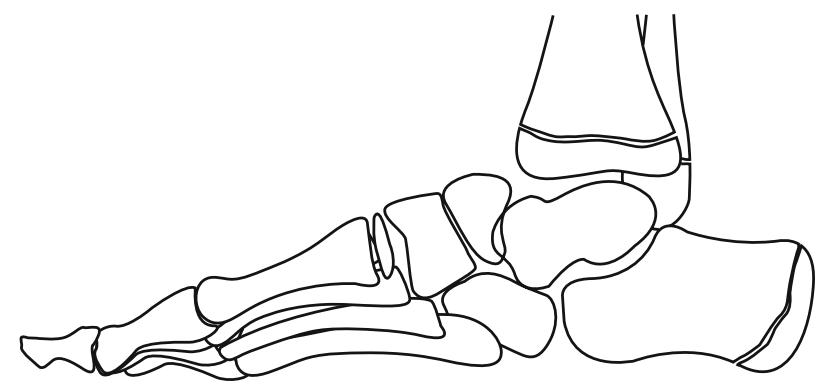

A

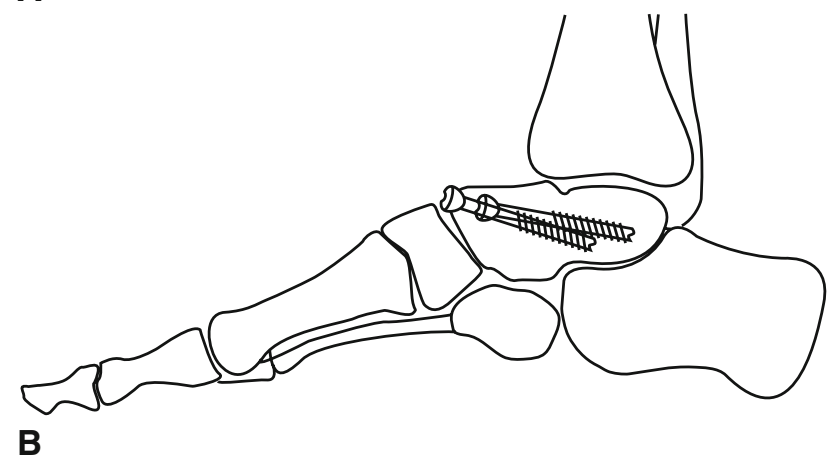

Fig. 1A-B (A) Preoperative standing lateral demonstrating dorsal rotatory subluxation of the navicular on the head of the talus. Note also the loss of normal calcaneal pitch as compared to the contralateral side. (B) Postoperative standing lateral radiograph demonstrating talonavicular arthrodesis with restoration of calcaneal pitch. (range, $0-16^{\circ}$ ). The AP talo-first metatarsal angle changed only minimally, from a preoperative average of $16^{\circ}$ (range, $3^{\circ}-46^{\circ}$ ) to postoperative average of $14^{\circ}$ (range, $0-39^{\circ}$ ) (Fig. 1A-B).

One patient initially treated by another surgeon with attempted talonavicular arthrodesis developed nonunion of the talonavicular joint. We subsequently attempted revision arthrodesis. The patient developed a pseudarthrosis and then underwent a third fusion using autogenous iliac crest bone graft. After this procedure the patient developed bony fusion. All of the other operated feet went on to achieve bony fusion uneventfully, and no other patients developed complications. Two feet $(15 \%)$ underwent additional surgery for removal of symptomatic hardware.

\section{Discussion}

Dorsal rotatory subluxation of the navicular commonly follows the surgical treatment of resistant clubfeet. The subluxation typically progresses after posteromedial release. Patients develop a cavovarus foot deformity and have pain, abnormal gait, and difficulty with shoe wear. Previous attempts at soft tissue release and reduction have had limited success. We therefore reviewed our patients with persistent dorsal rotatory subluxation of the navicular treated with talonavicular arthrodesis. We described the results of arthrodesis in terms of symptomatic relief, radiographic correction, and associated complications.

This study is limited by a small number of patients and relatively short followup period. However, we present the series in order to highlight the difficulties associated with treatment of persistent navicular subluxation and propose a treatment method which has been successful in the shortterm. Longer-term followup will be necessary to fully assess the results of talonavicular fusion.

Dorsal rotatory subluxation of the navicular is a common cause of progressive cavovarus foot deformity after posteromedial release for clubfoot (Fig. 2A-D). The clinical examination of a patient with dorsal rotatory subluxation demonstrates cavovarus of the forefoot with plantarflexion of the metatarsal heads. This represents breakdown through Chopart's joint, which may be related to a stiffened subtalar joint resulting from the initial operative intervention. When examining these patients, it is important to be aware that the heel may be in either varus or valgus depending on whether the foot has been previously over- or undercorrected. As a result, a sliding calcaneal osteotomy may be necessary in addition to treatment for the subluxated navicular in order to fully correct the foot position.

The etiology of dorsal rotatory subluxation of the navicular is not completely understood. Wei et al. propose 

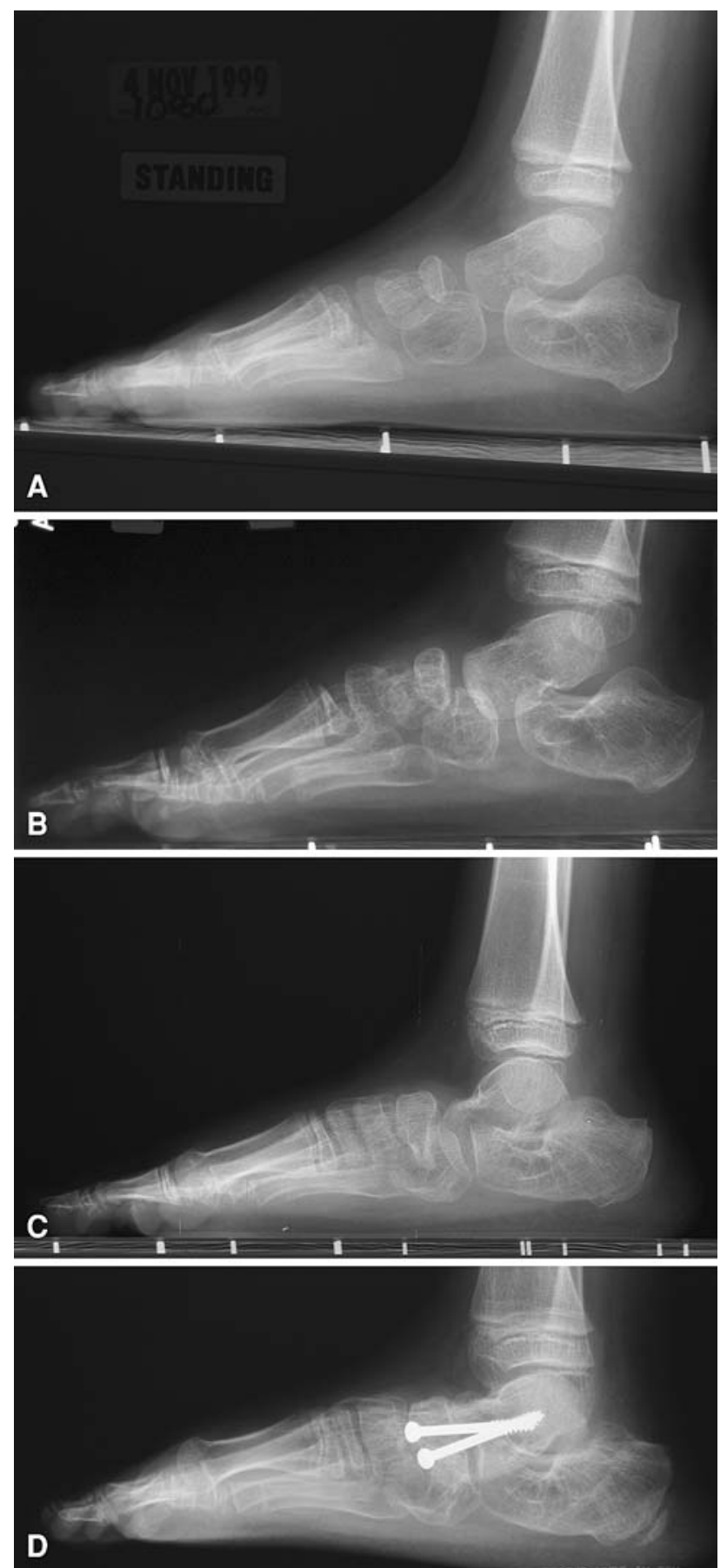

Fig. 2A-D Serial radiographs of a patient included in this series demonstrating progressive nature of cavovarus deformity and subluxation of the navicular. (A) Standing lateral radiograph of the patient at 3 years of age. Patient has previously undergone posteromedial release. (B) Standing lateral radiograph of the patient at 6 years of age. Note progression of subluxation with resulting increase in talo-first metatarsal angle. (C) Standing lateral radiograph of the patient at 10 years of age. Again noted is increased subluxation of the navicular, increase in talo-first metatarsal angle, and progressive loss of calcaneal pitch. (D) Standing lateral radiograph of the patient 1 year after talonavicular arthrodesis. Note correction of subluxation and normalization of talo-first metatarsal angle. that the deformity "results from excessive dorsally directed forces at the talonavicular joint in patients with no dorsiflexion at the ankle secondary to a flattop talus" [8]. Lau et al. state that release of the spring ligament with inaccurate pinning may be a contributing factor and stress the importance of direct visualization during the pinning process [5]. In agreement, Davidson stresses that since the navicular is not yet ossified at the time of initial clubfoot release, the surgeon cannot rely on intraoperative radiographs and should instead directly visualize the talonavicular joint during pinning. He suggests holding the foot in mild equinus during pinning to prevent unrecognized dorsal subluxation of the navicular [3].

Kuo and Jansen [4] performed an anatomic study in order to better delineate the nature of the deformity. They noted superior rotation of the medial end of the navicular together with an intact naviculocuneiform joint caused an increased cavus due to increased height of the medial arch. In addition, they proposed the increased varus and supination of the forefoot was caused by shortening and rotation of the medial arch while the normal lateral arch was maintained. They also suggested the unreleased lateral structure of the navicular was a tethering factor after extensive medial release and the ball-and-socket shape of the talonavicular joint facilitated a rotatory displacement. Although they observed no association between type of treatment of the posterior tibial tendon and development of dorsal rotatory subluxation, the authors suggested an unopposed anterior tibial tendon may pull the forefoot into supination and force rotation of the navicular.

Treatment of this complex deformity is challenging. Kuo and Jansen [4] believed correction required a complete midtarsal joint release with derotation of the navicular and pin fixation, however they did not report on any case examples or provide any followup data. We have also attempted to treat dorsal rotatory subluxation of the navicular with comprehensive soft tissue releases without success. Similarly, Barnett [1] described poor results with attempts at correction by repeat soft tissue release and reduction resulting in persistence of subluxation. Schlafly et al. [7] described a failed attempt to perform open reduction of a dorsally subluxated navicular in a previously treated clubfoot. They could not achieve reduction and subsequently state, "corrective surgery for postoperative dorsal navicular subluxation is not recommended" [7].

Barnett [1] has described the "Third Street Operation" in conjunction with a complete peritalar release as a proposed treatment for dorsal subluxation of the navicular. $\mathrm{He}$ suggested release was required to free the medial column of the foot from the lateral column in order to reduce the navicular. This procedure involves complete capsulotomy between the cuboid and navicular, cuboid and third 
cuneiform, and between the base of the third and fourth metatarsals. However this was described simply as a potential new technique and no report on results was given.

Wei et al. [8] previously reported 15 patients with either dorsolateral subluxation of the talonavicular joint, triangular navicular, and/or degenerative changes of the talonavicular joint who underwent 17 talonavicular arthrodeses. Fourteen of their 15 patients were completely satisfied with the procedure. One patient was partially satisfied due to development of naviculocuneiform arthritis 3 years after surgery. Fusion was achieved using either Kirschner wires (13 cases), screws (three cases), or staples (three cases). They reported three cases of nonunion, two of which underwent revision surgery. They stated that talonavicular fusion led to correction of the residual midfoot deformity citing improvement in talo-first metatarsal angle and the AP talo-first metatarsal angle.

The operation we describe provided a reliable method for achieving a symptom-free result with a single surgery. Similar to Wei et al. [8], we noted an improvement in talofirst metatarsal angle as a result of the arthrodesis. In addition, we observed a restoration in calcaneal pitch postoperatively (Fig. 1A-B). Also, the percent subluxation of the navicular decreased from $41.8 \%$ to $6 \%$ after fusion. This decrease in subluxation corrects the position of the foot, removes first ray pressure, and allows ease of shoe wear and improved cosmesis.

At final followup, 12 of the 13 patients in this series were symptom-free. Three patients with interest in returning to sports did so without difficulty. One of the patients initially treated by another surgeon developed nonunion; however, in the 12 patients initially operated on by the senior authors, there were no incidences of nonunion. The authors recommend placing two screws as was performed towards the end of the study period in order to achieve better compression, prevent rotation, and decrease the risk for nonunion.

Long-term followup of these patients is not known. What is the effect of motion now occurring through the navicular-cuneiform joints rather than talonavicular? We have encountered two patients with congenital talonavicular fusion who have excellent foot position, good motion, and no symptoms. Whether this will hold true for multiply operated clubfeet with stiff subtalar joints and this fusion remains to be seen.

Dorsal rotatory subluxation of the navicular as sequelae of previously treated clubfoot can lead to symptomatic cavovarus deformity of the foot. Previous attempts at open reduction and pinning have been unsuccessful. Talonavicular arthrodesis is an option for correcting the underlying cavovarus deformity associated with dorsal rotatory subluxation of the navicular and alleviating the accompanying symptoms of the plantar flexed first ray in a single surgical setting.

Open Access This article is distributed under the terms of the Creative Commons Attribution Noncommercial License which permits any noncommercial use, distribution, and reproduction in any medium, provided the original author(s) and source are credited.

\section{References}

1. Barnett RM Sr. Medial/lateral column separation (third street operation) for dorsal talonavicular subluxation. In: Simons GW, ed. The Clubfoot: The Present and View of the Future. New York, NY: Springer-Verlag; 1994:268-272.

2. Blakeslee TJ, DeValentine SJ. Management of the resistant idiopathic clubfoot: the Kaiser experience from 1980-1990. J Foot Ankle Surg. 1995:34:167-176.

3. Davidson RS. Operative treatment of resistant clubfoot. In: Tolo VT, Skaggs DL, eds. Master Techniques in Orthopaedic Surgery: Pediatrics. Philadelphia, PA: Lippincott Williams \& Wilkins; 2008:211-219.

4. Kuo KN, Jansen LD. Rotatory dorsal subluxation of the navicular: A complication of clubfoot surgery. $J$ Pediatr Orthop. 1998;18:770-774.

5. Lau JHK, Meyer LC, Lau HC. Results of surgical treatment of talipes equinovarus congenital. Clin Orthop Relat Res. 1989;248:219-226.

6. Miller JH, Bernstein SM. The roentgenographic appearance of the "corrected clubfoot." Foot Ankle. 1986;6:177-183.

7. Schlafly B, Butler JE, Siff SJ, Criswell AR, Cain TE. The appearance of the tarsal navicular after posteromedial release for clubfoot. Foot Ankle. 1985;5:222-237.

8. Wei SY, Sullivan RJ, Davidson RS. Talo-navicular arthrodesis for residual midfoot deformities of a previously corrected clubfoot. Foot Ankle Int. 2000;21:482-485. 